\title{
Soliton Solutions and Collisions for the Multicomponent Gross-Pitaevskii Equation in Spinor Bose-Einstein Condensates
}

\author{
Ming Wang (i) and Guo-Liang He \\ School of Mathematics and Information Science, Zhengzhou University of Light Industry, Zhengzhou, Henan 450002, China \\ Correspondence should be addressed to Ming Wang; hsxh1943@126.com
}

Received 29 March 2020; Revised 1 June 2020; Accepted 23 June 2020; Published 31 July 2020

Guest Editor: Cuimei Jiang

Copyright (c) 2020 Ming Wang and Guo-Liang He. This is an open access article distributed under the Creative Commons Attribution License, which permits unrestricted use, distribution, and reproduction in any medium, provided the original work is properly cited.

In this paper, we investigate a five-component Gross-Pitaevskii equation, which is demonstrated to describe the dynamics of an $F=2$ spinor Bose-Einstein condensate in one dimension. By employing the Hirota method with an auxiliary function, we obtain the explicit bright one- and two-soliton solutions for the equation via symbolic computation. With the choice of polarization parameter and spin density, the one-soliton solutions are divided into four types: one-peak solitons in the ferromagnetic and cyclic states and one- and two-peak solitons in the polar states. For the former two, solitons share the similar shape of one peak in all components. Solitons in the polar states have the one- or two-peak profiles, and the separated distance between two peaks is inversely proportional to the value of polarization parameter. Based on the asymptotic analysis, we analyze the collisions between two solitons in the same and different states.

\section{Introduction}

Bose-Einstein condensates (BECs) of the alkali-metalatom gases have attracted certain attention in both experimental $[1,2]$ and theoretical studies [3-5]. BECs can have the internal degrees of freedom associated with the hyperfine spin and such condensates are usually called the spinor BECs [6]. The spinor BECs are classified according to the relative values of certain characteristic scattering lengths [7]. For the spinor BEC with the hyperfine spin $F=1$, the condensate can have either ferromagnetic state and polar state, such as ${ }^{87} \mathrm{Rb}$ and ${ }^{23} \mathrm{Na}$, respectively, and the corresponding ground-state structure and dynamical properties of those two cases are distinct [8]. In the description of $[7,8]$, a spinor condensate with the spin $F=2$ bosons in an optical trap can be one of the three types: ferromagnetic, polar, and cyclic. Dynamics of the spinor BEC have been addressed in [9-14].

The dynamics of nonlinear phenomena can be analyzed by means of the corresponding nonlinear evolution equations. There has been considerable work carried out on the control problem of nonlinear systems, such as those in chaotic and stochastic systems [15-19]. Solitons, resulted from the balance between the effects of nonlinearity and dispersion [20], have been studied in fields such as nonlinear optics, plasma physics, and condensed matter physics [3, 21-23]. In addition, breathers and rogue waves have been concerned in recent research $[24,25]$. In the spinor BECs, matter-wave solitons are thought to be useful for their application in the atom laser, atom interferometry, and coherent atom transport, which can contribute to the realization of quantum information processors or computation [26]. The dynamics of magnetic soliton, dark soliton, and dark-bright vector soliton in spinor Bose-Einstein condensates have been investigated [27-30]. A frontier is the model extension from the single-component condensate to the multicomponent, which opens up new fields for the study of quantum matter waves and fluids [11]. The spinor BECs with spin $F$ is described by a $(2 F+1)$-component mean-field wave function [31].

In this paper, we will consider a five-component Gross-Pitaevskii (GP) equation for the dynamics of an $F=2$ spinor BEC in one dimension [32]: 


$$
\begin{aligned}
& i \partial_{t} \Phi_{ \pm 2}+\partial_{x}^{2} \Phi_{ \pm 2}+2 n \Phi_{ \pm 2}-\Theta \Phi_{\mp 2}^{*}=0, \\
& i \partial_{t} \Phi_{ \pm 1}+\partial_{x}^{2} \Phi_{ \pm 1}+2 n \Phi_{ \pm 1}+\Theta \Phi_{\mp 1}^{*}=0, \\
& i \partial_{t} \Phi_{0}+\partial_{x}^{2} \Phi_{0}+2 n \Phi_{0}-\Theta \Phi_{0}^{*}=0,
\end{aligned}
$$

where $\Phi_{ \pm j}$ 's $(j=0,1,2)$ are the wave functions of the five spin components, $t$ and $x$, respectively, denote the time and spatial co-ordinate, and the asterisk represents the complex conjugation, while the number density $n$ and singlet-pair amplitude $\Theta$ are defined by $[10,33]$

$$
\begin{aligned}
& n=\sum_{k=-2}^{2} \Phi_{k} \Phi_{k}^{*}, \\
& \Theta=2 \Phi_{2} \Phi_{-2}-2 \Phi_{1} \Phi_{-1}+\Phi_{0}^{2} .
\end{aligned}
$$

Equation (1) is the integrable version of the multicomponent GP equation mentioned in [31] and simplified under the condition $2 m=1, \hbar=1, c_{2}=0, c_{4}=1$, and $c_{0}=-2<0$, which corresponds to the attractive interaction. Due to the integrability, equation (1) has the infinite conservation laws, which restricts the dynamics of the system in an important way. The normalization is imposed as $\int d x n=N_{T}$, where $N_{T}$ is the total number of atoms. For equation (1), bright one- and two-soliton solutions have been given via the Zakharov-Shabat dressing [32]. However, the solitons are not classified according to the forms of the solutions and then the properties of soliton collisions are not clearly analyzed, that is the aim of this paper.

In this paper, we will concentrate on the soliton types and collisions in the same and different states in an $F=2$ spinor BEC. For equation (1), we will present the Hirota form with an auxiliary function and explicit one- and twosoliton solutions. With symbolic computation $[34,35]$, this paper will be organized as follows. In Section 2, the Hirota bilinearization procedure for equation (1) will be presented. In Section 3, based on the bilinear form, we will obtain and analyze the one-soliton solutions, which reveal the one- or two-peak solitons in three different states. In Section 4, making the asymptotic analysis on the obtained two-soliton solutions, we will discuss the soliton collisions in the same and different states. Section 5 will be our conclusions.

\section{Hirota Bilinearization Method for Equation (1)}

In order to understand the dynamics of equation (1), it is essential to obtain the soliton solutions. The Hirota bilinear method is a tool to construct the soliton solutions for certain nonlinear evolution equations [36]. For equation (1), we will utilize the Hirota method with an auxiliary function to obtain the one- and two-soliton solutions. The procedure can be extended to obtain $\mathrm{N}$-soliton solutions [37].

To begin with, equation (1) can be expressed in the bilinear form:

$$
\begin{aligned}
\left(i D_{t}+D_{x}^{2}\right)\left(G_{ \pm 2} \cdot F\right) & =S G_{\mp 2}^{*}, \\
\left(i D_{t}+D_{x}^{2}\right)\left(G_{ \pm 1} \cdot F\right) & =-S G_{\mp 1}^{*}, \\
\left(i D_{t}+D_{x}^{2}\right)\left(G_{0} \cdot F\right) & =S G_{0}^{*}, \\
D_{x}^{2}(F \cdot F) & =2 \sum_{k=-2}^{2} G_{k} G_{k}^{*}, \\
S F & =2 G_{2} G_{-2}-2 G_{1} G_{-1}+G_{0}^{2},
\end{aligned}
$$

with an auxiliary function $S$ and the following transformations:

$$
\Phi_{ \pm j}=\frac{G_{ \pm j}}{F}, \quad \text { for } j=0,1,2,
$$

where $G_{j}$ 's are the complex functions of $t$ and $x$ and $F$ is a real one. $D_{x}$ and $D_{t}$ are the bilinear differential operators [36] defined by

$$
D_{x}^{l} D_{t}^{m}(f \cdot g)=\left.\left(\frac{\partial}{\partial x}-\frac{\partial}{\partial x^{\prime}}\right)^{l}\left(\frac{\partial}{\partial t}-\frac{\partial}{\partial t^{\prime}}\right)^{m} f(x, t) g\left(x^{\prime}, t^{\prime}\right)\right|_{x^{\prime}=x, t^{\prime}=t} .
$$

where $l$ and $m$ are the positive integers and $x^{\prime}$ and $t^{\prime}$ are the formal variables. Multisoliton solutions for equation (1) can be generated by solving equation (3) with the following power series expansions as

$$
\begin{aligned}
G_{ \pm j} & =\varepsilon g_{ \pm j}^{(1)}+\varepsilon^{3} g_{ \pm j}^{(3)}+\varepsilon^{5} g_{ \pm j}^{(5)}+\cdots, \\
F & =1+\varepsilon^{2} f_{2}+\varepsilon^{4} f_{4}+\varepsilon^{6} f_{6}+\cdots, \\
S & =\varepsilon^{2} s_{2}+\varepsilon^{4} s_{4}+\varepsilon^{6} s_{6}+\cdots,
\end{aligned}
$$

where $\varepsilon$ is the formal parameter, $g_{ \pm j}^{(l)} s(l=1,3,5, \ldots)$ are the complex functions of $t$ and $x$, and $f_{m}$ 's $(m=2,4,6, \cdots)$ are the real ones which will be determined later.

\section{Bright One-Soliton Solutions}

In order to obtain one-soliton solutions for equation (1), the power series expansions for $G_{ \pm j}, F$, and $S$ are terminated as

$$
\begin{aligned}
G_{ \pm j} & =\varepsilon g_{ \pm j}^{(1)}+\varepsilon^{3} g_{ \pm j}^{(3)}, \\
S & =\varepsilon^{2} S_{2}, \\
F & =1+\varepsilon^{2} f_{2}+\varepsilon^{4} f_{4} .
\end{aligned}
$$

It is noted that equation (7) are usually taken to obtain the two-soliton solutions. Substituting equation (7) into equation (3) and collecting the terms with the same power of $\mathcal{E}$, we obtain the one-soliton solutions for equation (1):

$$
\Phi_{ \pm j}=\frac{b_{1}^{( \pm j)} e^{\theta_{1}}+e^{2 \theta_{1}+\theta_{1}^{*}+d_{1}^{( \pm j)}}}{1+e^{\theta_{1}+\theta_{1}^{*}+l_{1}}+e^{2 \theta_{1}+2 \theta_{1}^{*}+c_{11}}},
$$

and the auxiliary function $S$ given as

$$
S=\Lambda_{1} e^{2 \theta_{1}},
$$


with

$$
\begin{aligned}
\theta_{1} & =k_{1} x+i k_{1}^{2} t, \\
e^{d_{1}^{( \pm j)}} & =\frac{\delta(j) b_{1}^{(\mp j)^{*}} \Lambda_{1}}{2\left(k_{1}+k_{1}^{*}\right)^{2}}, \\
\delta(j) & =\left\{\begin{array}{cc}
-1 & j=1 \\
1 & j=0,2,
\end{array}\right. \\
e^{l_{1}} & =\frac{\sum_{k=0}^{2}\left|b_{1}^{( \pm k)}\right|^{2}}{\left(k_{1}+k_{1}^{*}\right)^{2}}, \\
e^{c_{11}} & =\frac{\left|\Lambda_{1}\right|^{2}}{4\left(k_{1}+k_{1}^{*}\right)^{4}}, \\
\Lambda_{1} & =2 b_{1}^{(+1)} b_{1}^{(-1)}+\left(b_{1}^{(0)}\right)^{2}-2 b_{1}^{(+2)} b_{1}^{(-2)},
\end{aligned}
$$

where $b_{1}^{( \pm j)}$, s and $k_{1}$ are all complex constants. Here, we give the spin densities $f=\left(f^{x}, f^{y}, f^{z}\right)$ with [32]

$$
\begin{aligned}
& f^{x}=2\left[\Phi_{-2} \Phi_{-1}^{*}+\frac{\sqrt{6}}{2} \Phi_{0}^{*}\left(\Phi_{+1}+\Phi_{-1}\right)+\Phi_{2} \Phi_{1}^{*}\right]_{R}, \\
& f^{y}=2 i\left[\Phi_{-2} \Phi_{-1}^{*}+\frac{\sqrt{6}}{2} \Phi_{0}^{*} \Phi_{-1}+\frac{\sqrt{6}}{2} \Phi_{0} \Phi_{1}^{*}+\Phi_{1} \Phi_{2}^{*}\right]_{I}, \\
& f^{z}=2\left|\Phi_{-2}\right|^{2}+\left|\Phi_{-1}\right|^{2}-\left|\Phi_{1}\right|^{2}-2\left|\Phi_{2}\right|^{2},
\end{aligned}
$$

where the subscripts $R$ and $I$, respectively, denote the real and imaginary parts and the total spin can be given with $\int d x f=F_{T}$. For equation (1), there exist different types of solitons according to the values of $\Lambda_{1}$. When $\Lambda_{1}=0$, equation (8) admit the one-peak solitons in the ferromagnetic and cyclic states corresponding to the spin densities $|f|>0$ and $f=0$, respectively. For the case of $\Lambda_{1} \neq 0$, we can find the one- and two-peak solitons in the polar state, while only the two-peak soliton in the polar state for the $F=1$ spinor BECs [38].

3.1. One-Peak Solitons in the Ferromagnetic and Cyclic States. When $\Lambda_{1}=0$, equation (9) can be expressed as

$$
\Phi_{ \pm j}=A_{ \pm j} e^{i \theta_{1 I}} \operatorname{sech}\left(\theta_{1 R}+\frac{l_{1}}{2}\right),
$$

with

$$
\begin{aligned}
A_{ \pm j} & =\frac{1}{2} b_{1}^{( \pm j)} e^{-\left(l_{1} / 2\right)}, \\
k_{1} & =k_{1 R}+i k_{1 I}, \\
\theta_{1 R} & =k_{1 R}\left(x-2 k_{1 I} t\right), \\
\theta_{1 I} & =k_{1 I} x+\left(k_{1 R}^{2}-k_{1 I}^{2}\right) t .
\end{aligned}
$$

Here, $A_{ \pm j}, 2 k_{I}$, and $l_{1} / 2 k_{R}$, respectively, characterize the amplitude, velocity, and initial phase of the soliton. For $|f|>0$, it corresponds to the one-peak soliton in the ferromagnetic state [31]. When choosing suitable parameters to make $f=0$, we find that the soliton is in the cyclic state. Those two types of solitons posses the similar intensity profile of one peak in all components, as shown in Figures 1 and 2. For simplicity, we choose the parameters to make $\left|\Phi_{+j}\right|=\left|\Phi_{-j}\right|$ in Figure 1. It is noted that the auxiliary function $S$ vanishes in this case, as given in equation (12).

3.2. One- and Two-Peak Solitons in the Polar State. For the case $\Lambda_{1} \neq 0$, equation (8) can be expressed as

$$
\Phi_{ \pm j}=A_{ \pm j} e^{i \theta_{1 I}} \frac{\cosh \left(\theta_{1 R}+\left(c_{11} / 4\right)+P_{ \pm j}\right)}{4 \cosh ^{2}\left(\theta_{1 R}+\left(c_{11} / 4\right)\right)+L},
$$

where $\quad A_{ \pm j}=2 e^{\left(d_{1}^{( \pm j)}+\ln b_{1}^{( \pm j)}-c_{11}\right) / 2}, \quad L=e^{\left(l_{1}-\left(c_{11} / 2\right)\right)}-2$, and $P_{ \pm j}=e^{\left(2 d_{1}^{( \pm j)}-2 \ln b_{1}^{( \pm j)}-c_{11}\right) / 4}$. The form of equation (14) will be of use in the following asymptotic analysis of the twosoliton solutions. When $f=0$ under certain constraint, equation (14) depicts the one-peak soliton in the polar state, as the short dashed lines shown in Figure 3. When $f \neq 0$ but the spin $F=\int f \mathrm{~d} x=0$, we can observe the twopeak soliton in the polar state, as the solid lines shown in Figure 3. In addition, as the value of $\Lambda_{1}$ decreases, the separated distance between two peaks increases, and it looks like that there exist two ferromagnetic solitons traveling parallel in the same velocity and amplitudes as $\Lambda_{1}$ approaches to zero, as the long dashed lines shown in Figure 3.

\section{Two-Soliton Solutions and Soliton Collisions}

In this paper, we will concentrate on the two-soliton solutions and analyze the collisions between two solitons. Employing the following expansions,

$$
\begin{aligned}
G_{ \pm j} & =\varepsilon g_{ \pm j}^{(1)}+\varepsilon^{3} g_{ \pm j}^{(3)}+\varepsilon^{5} g_{ \pm j}^{(5)}+\varepsilon^{7} g_{ \pm j}^{(7)} \\
S & =\varepsilon^{2} s_{2}+\varepsilon^{4} s_{4}+\varepsilon^{6} s_{6} \\
F & =1+\varepsilon^{2} f_{2}+\varepsilon^{4} f_{4}+\varepsilon^{6} f_{6}+\varepsilon^{8} f_{8}
\end{aligned}
$$

We obtain the two-soliton solutions for equation (1) in the form of

$$
\Phi_{ \pm j}=\frac{G_{ \pm j}}{F}, \quad j=0,1,2,
$$

where the functions $G_{ \pm j}$ and $F$ are expressed as 


$$
\begin{aligned}
G_{ \pm j}= & b_{1}^{( \pm j)} e^{\theta_{1}}+b_{2}^{( \pm j)} e^{\theta_{2}}+e^{2 \theta_{1}+\theta_{1}^{*}+d_{1}^{( \pm j)}}+e^{2 \theta_{1}+\theta_{2}^{*}+d_{12}^{( \pm j)}}+e^{2 \theta_{2}+\theta_{1}^{*}+d_{21}^{( \pm j)}} \\
& +e^{2 \theta_{2}+\theta_{2}^{*}+d_{2}^{( \pm j)}}+e^{\theta_{1}+\theta_{1}^{*}+\theta_{2}+D_{12}^{( \pm j)}}+e^{\theta_{2}+\theta_{2}^{*}+\theta_{1}+D_{21}^{( \pm j)}}+e^{2 \theta_{1}+2 \theta_{1}^{*}+\theta_{2}+h_{12}^{( \pm j)}}+e^{2 \theta_{2}+2 \theta_{2}^{*}+\theta_{1}+h_{21}^{( \pm j)}}+e^{2 \theta_{2}+2 \theta_{1}^{*}+\theta_{1}+H_{12}^{( \pm j)}} \\
& +e^{2 \theta_{1}+2 \theta_{2}^{*}+\theta_{2}+H_{21}^{( \pm j)}}+e^{2 \theta_{1}+\theta_{1}^{*}+\theta_{2}+\theta_{2}^{*}+w_{12}^{( \pm j)}}+e^{2 \theta_{2}+\theta_{2}^{*}+\theta_{1}+\theta_{1}^{*}+w_{21}^{( \pm j)}}+e^{2 \theta_{1}+2 \theta_{1}^{*}+2 \theta_{2}+\theta_{2}^{*}+W_{12}^{( \pm j)}}+e^{2 \theta_{1}+2 \theta_{2}+2 \theta_{2}^{*}+\theta_{1}^{*}+W_{21}^{( \pm j)}} \\
F= & 1+e^{\theta_{1}+\theta_{1}^{*}+l_{1}}+e^{\theta_{1}+\theta_{2}^{*}+l_{0}}+e^{\theta_{2}+\theta_{1}^{*}+l_{0}^{*}}+e^{\theta_{2}+\theta_{2}^{*}+l_{2}}+e^{2 \theta_{1}+2 \theta_{1}^{*}+c_{11}}+e^{2 \theta_{1}+2 \theta_{2}^{*}+c_{12}}+e^{2 \theta_{2}+2 \theta_{1}^{*}+c_{12}^{*}}+e^{2 \theta_{2}+2 \theta_{2}^{*}+c_{22}} \\
& +e^{2 \theta_{1}+\theta_{1}^{*}+\theta_{2}^{*}+l_{12}}+e^{2 \theta_{1}^{*}+\theta_{1}+\theta_{2}+l_{12}^{*}}+e^{2 \theta_{2}+\theta_{1}^{*}+\theta_{2}^{*}+l_{21}}+e^{2 \theta_{2}^{*}+\theta_{1}+\theta_{2}+l_{21}^{*}}+e^{\theta_{1}+\theta_{1}^{*}+\theta_{2}+\theta_{2}^{*}+l_{3}}+e^{2 \theta_{1}+2 \theta_{1}^{*}+\theta_{2}+\theta_{2}^{*}+L_{12}} \\
& +e^{2 \theta_{2}+2 \theta_{2}^{*}+\theta_{1}+\theta_{1}^{*}+L_{21}}+e^{2 \theta_{1}+2 \theta_{2}^{*}+\theta_{2}+\theta_{1}^{*}+l_{4}}+e^{2 \theta_{2}+2 \theta_{1}^{*}+\theta_{1}+\theta_{2}^{*}+l_{4}^{*}}+e^{2 \theta_{1}+2 \theta_{1}^{*}+2 \theta_{2}+2 \theta_{2}^{*}+l_{5}}
\end{aligned}
$$

and the auxiliary function $S$ takes the form

with

$$
\begin{aligned}
S= & \Lambda_{1} e^{2 \theta_{1}}+\Lambda_{2} e^{2 \theta_{2}}+e^{\theta_{1}+\theta_{2}+r_{1}}+e^{\theta_{1}+\theta_{1}^{*}+2 \theta_{2}+r_{21}}+e^{\theta_{2}+\theta_{2}^{*}+2 \theta_{1}+r_{12}} \\
& +e^{\theta_{1}+\theta_{2}^{*}+2 \theta_{2}+R_{21}}+e^{\theta_{2}+\theta_{1}^{*}+2 \theta_{1}+R_{12}}+e^{2 \theta_{1}+2 \theta_{1}^{*}+2 \theta_{2}+r_{3}} \\
& +e^{2 \theta_{1}+2 \theta_{2}+2 \theta_{2}^{*}+r_{4}}+e^{2 \theta_{1}+\theta_{1}^{*}+2 \theta_{2}+\theta_{2}^{*}+r_{5}},
\end{aligned}
$$

$$
\begin{aligned}
& \theta_{m}=k_{m} x+i k_{m}^{2} t \\
& \Lambda_{m}=2 b_{m}^{(+1)} b_{m}^{(-1)}+\left(b_{m}^{(0)}\right)^{2}-2 b_{m}^{(+2)} b_{m}^{(-2)}, \\
& m=1,2 \text {, } \\
& e^{d_{m}^{( \pm j)}}=\frac{\delta(j) b_{m}^{(\mp j) *} \Lambda_{m}}{2\left(k_{m}+k_{m}^{*}\right)^{2}} \\
& e^{d_{l m}^{( \pm j)}}=\frac{\delta(j) b_{l}^{(\mp j) *} \Lambda_{m}}{\left(k_{m}+k_{l}^{*}\right)^{2}} \\
& l=1,2, m \neq l, \delta(j)= \begin{cases}-1, & j=1, \\
1, & j=0,2,\end{cases} \\
& e^{D_{l m}^{( \pm j)}}=\frac{1}{\left(k_{m}+k_{m}^{*}\right)^{2}\left(k_{l}+k_{m}^{*}\right)^{2}}\left\{2\left(k_{m}-k_{l}\right)\left[b_{m}^{( \pm j)}\left(k_{m}+k_{m}^{*}\right) \sum_{k=0}^{2} b_{m}^{( \pm k) *} b_{l}^{( \pm k)}-b_{l}^{( \pm j)}\left(k_{l}+k_{m}^{*}\right) \sum_{k=0}^{2} \delta(k)\left|b_{m}^{( \pm k)}\right|^{2}\right]\right. \\
& \left.+\delta(j) b_{m}^{(\mp j) *}\left(k_{m}+k_{m}^{*}\right)\left(k_{l}+k_{m}^{*}\right) \sum_{k=0}^{2} \delta(k) b_{m}^{( \pm k)} b_{l}^{( \pm k)}\right\} \\
& e^{h_{l m}^{( \pm j)}}=\frac{b_{l}^{( \pm j)}\left|\Lambda_{m}\right|^{2}\left(k_{m}^{*}-k_{l}^{*}\right)^{2}}{4\left(k_{m}+k_{m}^{*}\right)^{4}\left(k_{l}+k_{m}^{*}\right)^{2}} \\
& e^{H_{l m}^{( \pm j)}}=\frac{b_{m}^{(\mp j) *} \Lambda_{m} \Lambda_{l}^{*}\left(k_{m}^{*}-k_{l}^{*}\right)^{2}}{4\left(k_{m}+k_{m}^{*}\right)^{2}\left(k_{m}+k_{l}^{*}\right)^{4}}
\end{aligned}
$$




$$
\begin{aligned}
& e^{w_{l m}^{( \pm)}}=\frac{\Lambda_{m}\left(k_{m}-k_{l}\right)^{2}}{2\left(k_{m}+k_{m}^{*}\right)^{2}\left|k_{m}+k_{l}^{*}\right|^{4}\left(k_{l}+k_{l}^{*}\right)^{2}}\left\{b_{l}^{( \pm j)}\left(k_{l}+k_{l}^{*}\right)\left(k_{l}+k_{m}^{*}\right) \sum_{k=0}^{2} \delta(k) b_{m}^{(\mp k) *} b_{l}^{( \pm k) *}+\delta(j)\left(k_{m}^{*}-k_{l}^{*}\right)\right. \\
& \left.\left[b_{m}^{(\mp j) *}\left(k_{l}+k_{m}^{*}\right) \sum_{k=0}^{2}\left|b_{l}^{( \pm k)}\right|^{2}-b_{l}^{(\mp j) *}\left(k_{l}+k_{l}^{*}\right) \sum_{k=0}^{2} b_{m}^{( \pm k) *} b_{l}^{( \pm k)}\right]\right\} \text {, } \\
& e^{W_{l m}^{( \pm)}}=\frac{\delta(j) b_{l}^{(\mp j) *}\left|\Lambda_{m}\right|^{2} \Lambda_{l}\left(k_{m}-k_{l}\right)^{4}\left(k_{m}^{*}-k_{l}^{*}\right)^{2}}{8\left(k_{m}+k_{m}^{*}\right)^{4}\left(k_{l}+k_{m}^{*}\right)^{4}\left(k_{m}+k_{l}^{*}\right)^{2}\left(k_{l}+k_{l}^{*}\right)^{2}}, \\
& e^{l_{0}}=\frac{\sum_{k=0}^{2} b_{1}^{( \pm k)} b_{2}^{( \pm k) *}}{\left(k_{1}+k_{2}^{*}\right)^{2}} \\
& e^{l_{m}}=\frac{\sum_{k=0}^{2}\left|b_{m}^{( \pm k)}\right|^{2}}{\left(k_{m}+k_{m}^{*}\right)^{2}} \\
& e^{c_{l m}}=\frac{\Lambda_{m} \Lambda_{l}^{*}}{4\left(k_{m}+k_{l}^{*}\right)^{4}} \\
& e^{l_{3}}=\frac{2}{\left(k_{1}+k_{1}^{*}+k_{2}+k_{2}^{*}\right)^{2}}\left[\left(\left|k_{1}\right|^{2}+\left|k_{2}\right|^{2}-2 k_{1}^{*} k_{2}\right)\left(\left|e^{c_{0}}\right|^{2}-e^{c_{1}} e^{c_{2}}\right)-\left(k_{1}^{2}+k_{2}^{2}-2 k_{1} k_{2}\right)\left(\left|e^{c_{0}}\right|^{2}+e^{c_{1}} e^{c_{2}}\right)-2 \sum_{m, l=1, m \neq l}^{2} \sum_{k=0}^{2} b_{m}^{( \pm k) *} e^{D_{l m}^{(k)}}\right], \\
& e^{l_{l m}}=\frac{\Lambda_{m} \sum_{k=0}^{2} \delta(k) b_{1}^{(\mp k) *} b_{2}^{( \pm k) *}}{2\left(k_{m}+k_{m}^{*}\right)^{2}\left(k_{m}+k_{l}^{*}\right)^{2}} \\
& e^{L_{l m}}=\frac{\left|\Lambda_{m}\right|^{2} \sum_{k=0}^{2}\left|b_{l}^{( \pm k)}\right|^{2}\left|k_{1}-k_{2}\right|^{4}}{4\left(k_{m}+k_{m}^{*}\right)^{4}\left|k_{m}+k_{l}\right|^{4}\left(k_{l}+k_{l}^{*}\right)^{2}}, \\
& e^{l_{4}}=\frac{\Lambda_{1} \Lambda_{2}^{*} \sum_{k=0}^{2} b_{2}^{( \pm k)} b_{1}^{( \pm k) *}\left|k_{1}-k_{2}\right|^{4}}{4\left(k_{1}+k_{1}^{*}\right)^{2}\left|k_{1}+k_{2}\right|^{4}\left(k_{1}+k_{2}^{*}\right)^{2}\left(k_{2}+k_{2}^{*}\right)^{2}} \\
& e^{m_{5}}=\frac{\left|\Lambda_{1}\right|^{2}\left|\Lambda_{2}\right|^{2}\left|k_{1}-k_{2}\right|^{8}}{16\left(k_{1}+k_{1}^{*}\right)^{4}\left|k_{1}+k_{2}^{*}\right|^{8}\left(k_{2}+k_{2}^{*}\right)^{4}} \text {, } \\
& e^{r_{1}}=2 \sum_{k=0}^{2} \delta(k) b_{1}^{(\mp k)} b_{2}^{( \pm k)} \text {, } \\
& e^{r_{l m}}=\frac{\Lambda_{m} \sum_{k=0}^{2}\left|b_{l}^{( \pm k)}\right|^{2}\left(k_{m}-k_{l}\right)^{2}}{\left(k_{m}+k_{l}^{*}\right)^{2}\left(k_{l}+k_{l}^{*}\right)^{2}}, \\
& e^{R_{l m}}=\frac{\Lambda_{m} \sum_{k=0}^{2} b_{l}^{( \pm k)} b_{m}^{( \pm k) *}\left(k_{1}-k_{2}\right)^{2}}{\left(k_{l}+k_{m}^{*}\right)^{2}\left(k_{m}+k_{m}^{*}\right)^{2}} \\
& e^{r_{3}}=\frac{\Lambda_{2}\left|\Lambda_{1}\right|^{2}\left(k_{1}-k_{2}\right)^{4}}{4\left(k_{1}+k_{1}^{*}\right)^{4}\left(k_{2}+k_{1}^{*}\right)^{4}} \\
& e^{r_{4}}=\frac{\Lambda_{1}\left|\Lambda_{2}\right|^{2}\left(k_{1}-k_{2}\right)^{4}}{4\left(k_{2}+k_{2}^{*}\right)^{4}\left(k_{1}+k_{2}^{*}\right)^{4}}, \\
& e^{r_{5}}=\frac{\Lambda_{1} \Lambda_{2} \sum_{k=0}^{2} \delta(k) b_{1}^{(\mp k) *} b_{2}^{( \pm k) *}\left(k_{1}-k_{2}\right)^{4}}{2\left(k_{1}+k_{1}^{*}\right)^{2}\left|k_{1}+k_{2}^{*}\right|^{4}\left(k_{2}+k_{2}^{*}\right)^{2}},
\end{aligned}
$$

where $k_{1}, k_{2}, b_{1}^{( \pm j)}$ 's, and $b_{2}^{( \pm j)}$, s are all complex constants. To understand the collision property of solitons in the $F=2$ spinor BEC, we will make the asymptotic analysis on two- soliton solution (16) as $t \longrightarrow \mp \infty$, which are associated with the initial and final state, respectively. Without loss of generality, we set 


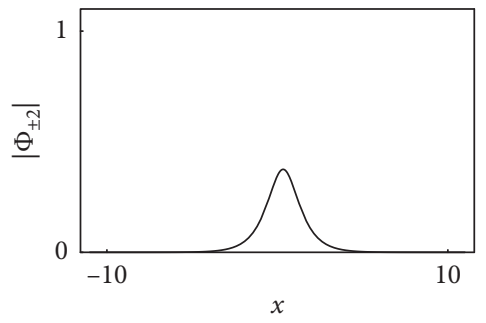

(a)

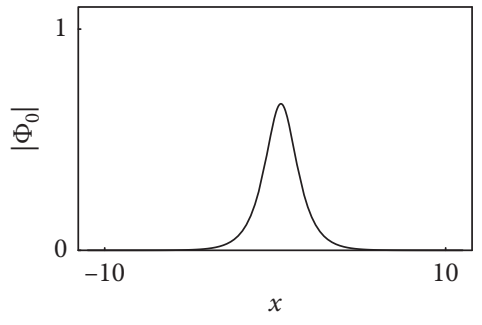

(b)

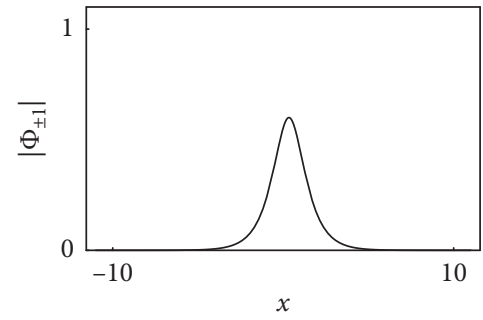

(c)

FIGURE 1: Intensity plots of the one soliton in the ferromagnetic state for the components $\Phi_{ \pm j}$ via solution (8). Parameters are $k_{1}=1.2+i$, $b_{1}^{( \pm 2)}=0.5, b_{1}^{(0)}=0.5$, and $b_{1}^{( \pm 1)}=\sqrt{0.78}$.

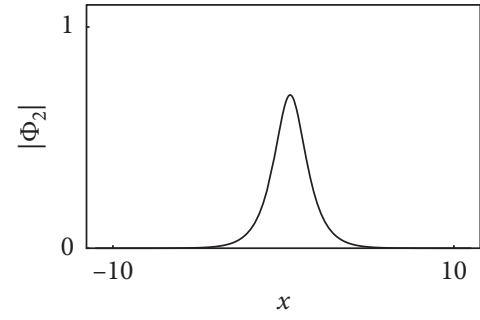

(a)

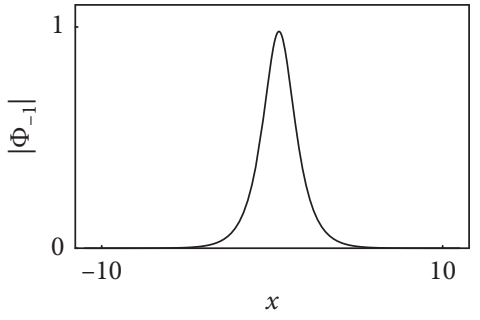

(b)

Figure 2: Intensity plots of the one soliton in the cyclic state for the components $\Phi_{2}$ and $\Psi_{-1}$ via solution (8). Parameters are $k_{1}=1.2+i$, $b_{1}^{(2)}=0.5+0.7 i, b_{1}^{(-2)}=b_{1}^{(0)}=b_{1}^{(1)}=0$, and $b_{1}^{(-1)}=\sqrt{2}(0.5-0.7 i)$.

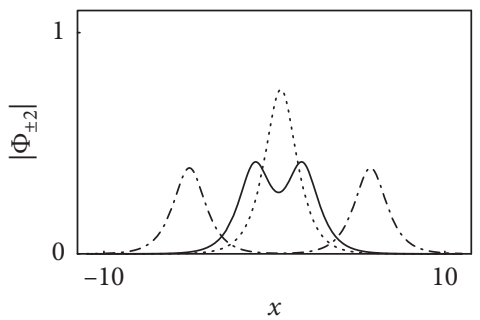

(a)

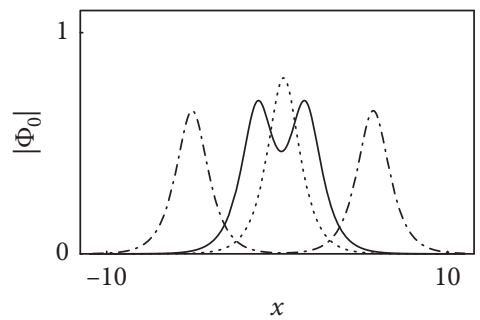

(b)

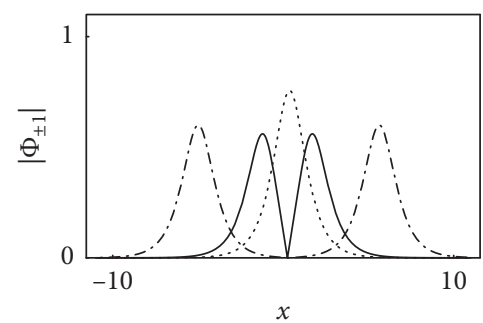

(c)

FIGURE 3: Intensity plots of the one soliton in the polar state for the components $\Phi_{ \pm j}$ via Solutions (8). Parameters are $k_{1}=1.2+i$ with (1) $b_{1}^{(2)}=b_{1}^{(-2) *}=0.5+0.8 i, b_{1}^{(0)}=1.01$, and $b_{1}^{(1)}=-b_{1}^{(-1) *}=0.75+0.6 i$, short dashed lines; $(2) b_{1}^{( \pm 2)}=0.6, b_{1}^{(0)}=1$, and $b_{1}^{( \pm 1)}=\sqrt{0.75}$, solid lines; (3) $b_{1}^{( \pm 2)}=0.6, b_{1}^{(0)}=1$, and $b_{1}^{( \pm 1)}=\sqrt{0.85999}$, long dashed lines.

$$
\begin{aligned}
& k_{1 R}>0, \\
& k_{1 I}>0, \\
& k_{2 R}>0, \\
& k_{2 I}<0 .
\end{aligned}
$$

Following the classification on the one-soliton solutions, we naturally consider the collisions between the same and different types of solitons, which are determined by $\Lambda_{m}$ and $f$. Since the one-peak soliton in the cyclic state is obtained under certain strong restriction, its collisions with other types of soliton are omitted here.

4.1. Collisions between Two Solitons in the Ferromagnetic State. At first, we will consider the collision between two solitons in the ferromagnetic state. Under the condition $\Lambda_{m}=0$, the asymptotic forms for equation (16) are given as follows:

(A) Before the collision $(t \longrightarrow-\infty)$.

(1) Soliton $S^{1-}\left(\theta_{1 R} \sim 0, \theta_{2 R} \longrightarrow-\infty\right)$ :

$$
\Phi_{ \pm j} \longrightarrow A_{ \pm j}^{1-} e^{i \theta_{1 I}} \operatorname{sech}\left(\theta_{1 R}+\frac{l_{1}}{2}\right) .
$$

(2) Soliton $S^{2-}\left(\theta_{2 R} \sim 0, \theta_{1 R} \longrightarrow \infty\right)$ :

$$
\Phi_{ \pm j} \longrightarrow A_{ \pm j}^{2-} e^{i \theta_{2 I}} \operatorname{sech}\left(\theta_{2 R}+\frac{l_{3}-l_{1}}{2}\right) .
$$


(B) After the collision $(t \longrightarrow+\infty)$.

(1) Soliton $S^{1+}\left(\theta_{1 R} \sim 0, \theta_{2 R} \longrightarrow \infty\right)$ :

$$
\Phi_{ \pm j} \longrightarrow A_{ \pm j}^{1+} e^{i \theta_{1 I}} \operatorname{sech}\left(\theta_{1 R}+\frac{l_{3}-l_{2}}{2}\right)
$$

(2) Soliton $S^{2+}\left(\theta_{2 R} \sim 0, \theta_{1 R} \longrightarrow-\infty\right)$ :

$$
\Phi_{ \pm j} \longrightarrow A_{ \pm j}^{2+} e^{i \theta_{2 I}} \operatorname{sech}\left(\theta_{2 R}+\frac{l_{2}}{2}\right)
$$

with

$$
\begin{aligned}
& A_{ \pm j}^{1-}=\frac{b_{1}^{( \pm j)}}{2} e^{-\left(l_{1} / 2\right)}, \\
& A_{ \pm j}^{2-}=\frac{1}{2} e^{\left(\left(D_{12}^{( \pm j)}-l_{2}-l_{3}\right) / 2\right),} \\
& A_{ \pm j}^{1+}=\frac{1}{2} e^{\left(\left(D_{21}^{( \pm j)}-l_{2}-l_{3}\right) / 2\right),} \\
& A_{ \pm j}^{2+}=\frac{b_{2}^{( \pm j)}}{2} e^{-\left(l_{2} / 2\right)} .
\end{aligned}
$$

The superscripts $m \pm(m=1,2)$ represent the solitons indicated as $S_{1}$ and $S_{2}$ at $t \longrightarrow \pm \infty$. Setting $b_{1}^{( \pm j)} /$ $b_{2}^{( \pm j)}=$ constant, we find that the amplitudes $A_{ \pm j}^{m \pm}$ before and after the collision can be related through $A_{+j}^{1+j}=\left(A_{+j}^{1-}\left(k_{1}-\right.\right.$ $\left.\left.k_{2}\right)\left(k_{1}^{*}+k_{2}\right)\right) /\left(\left(k_{1}^{*}-k_{2}^{*}\right)\left(k_{1}+k_{2}^{*}\right)\right)$ and $A_{ \pm j}^{2+}=\left(A_{ \pm j}^{2-}\left(k_{1}^{*}-\right.\right.$ $\left.\left.k_{2}^{*}\right)\left(k_{1}^{*}+k_{2}\right)\right) /\left(\left(k_{1}-k_{2}\right)\left(k_{1}+k_{2}^{*}\right)\right)$, namely, $\left|A_{ \pm j}^{m+}\right|=\left|A_{ \pm j}^{m-}\right|$, which means that the collision is elastic in this case. Besides, the solitons $S_{1}$ and $S_{2}$, respectively, experience a phase shift of $\eta_{1}=\left(l_{3}-l_{1}-l_{2}\right) / 4 k_{1 R}=\ln \left[\left(k_{1}-k_{2}\right)\left(k_{1}^{*}-k_{2}^{*}\right) /\left(k_{1}^{*}+k_{2}\right)\right.$ $\left.\left(k_{1}+k_{2}^{*}\right)\right] / k_{1 R}$ and $\eta_{2}=-\eta_{1} k_{1 R} / k_{2 R}$ during the collision. For other choices of $b_{m}^{( \pm j)}$ satisfying $\Lambda_{m}=0$, we will take an example and give Figure 4 . The intensity of soliton $S_{2}$ in component $\Phi_{0}$ decreases while those of $S_{2}$ in components $\Phi_{ \pm 1}$ increase after the collision, as shown in Figures $4(\mathrm{~b})$ and 4(c). This process might be of value for the realization of soliton switching devices [39]. The similar changes of soliton intensities can be observed for soliton $S_{1}$. For this case, the collision is similar to that with an intensity redistribution among all components of each soliton in the multicomponent NLS equation [40].

\subsection{Collisions between Soliton in the Ferromagnetic State and} Solitons in the Polar State. We will analyze the collisions between soliton in the ferromagnetic state and one- and twopeak solitons in the polar state. Here, we choose $S_{1}$ to be the soliton in the ferromagnetic state $\left(\Lambda_{1}=0\right)$, and $S_{2}$ to be the soliton in the polar state corresponding to the condition $\Lambda_{2} \neq 0$. The asymptotic forms of solitons $S_{1}$ and $S_{2}$ are given as follows:

(A) Before the collision.
(1) Soliton $S^{1-}$ :

$$
\Phi_{ \pm j} \longrightarrow A_{ \pm j}^{1-} e^{i \theta_{1 I}} \operatorname{sech}\left(\theta_{1 R}+\frac{l_{1}}{2}\right),
$$

where $A_{ \pm j}^{1-}=(1 / 2) b_{1}^{( \pm j)} e^{-\left(l_{1} / 2\right)}$.

(2) Soliton $S^{2-}$ :

$$
\Phi_{ \pm j} \longrightarrow A_{ \pm j}^{2-} e^{i \theta_{2 I}} \frac{\cosh \left(\theta_{2 R}^{-}+P_{ \pm j}^{2-}\right)}{4 \cosh ^{2}\left(\theta_{2 R}^{-}\right)+L^{2-}},
$$

where $\quad A_{ \pm j}^{2-}=2 e^{\left(W_{21}^{( \pm j)}+D_{12}^{( \pm j)}-L_{21}-l_{1}\right) / 2}, \quad L^{2-}=$ $e^{\left(l_{3}-\left(\left(L_{21}+l_{1}\right) / 2\right)\right)}-2, \quad P_{ \pm j}^{2-}=e^{\left(2 W_{21}^{( \pm j)}-2 D_{12}^{( \pm j)}-L_{21}+l_{1}\right) / 4}, \quad$ and $\theta_{2 R}^{-}=\theta_{2 R}+\left(\left(L_{21}-l_{1}\right) / 4\right)$, with

$$
\begin{aligned}
k_{2} & =k_{2 R}+i k_{2 I}, \\
\theta_{2 I} & =k_{2 I} x+\left(k_{2 R}^{2}-k_{2 I}^{2}\right) t, \\
\theta_{2 R} & =k_{2 R} x-2 k_{2 R} k_{2 I} t .
\end{aligned}
$$

(B) After the collision.

(1) Soliton $S^{1+}$ :

$$
\Phi_{ \pm j} \longrightarrow A_{ \pm j}^{1+} e^{i \theta_{1 I}} \operatorname{sech}\left(\theta_{1 R}+\frac{L_{12}-c_{22}}{2}\right),
$$

where $A_{ \pm j}^{1+}=(1 / 2) e^{\left(2 h_{12}^{( \pm j)}-L_{12}-c_{22}\right) / 2}$

(2) Soliton $S^{2+}$ :

$$
\Phi_{ \pm j} \longrightarrow A_{ \pm j}^{2+} e^{i \theta_{2 I}} \frac{\cosh \left(\theta_{2 R}^{+}+P_{ \pm j}^{2+}\right)}{4 \cosh ^{2}\left(\theta_{2 R}^{+}\right)+L^{2+}},
$$

where $A_{ \pm j}^{2-}=2 e^{\left(d_{2}^{( \pm j)}+\ln b_{2}^{( \pm j)}-c_{22}\right) / 2}, L^{2-}=e^{\left(l_{2}-c_{22}\right) / 2}-2$, $P_{ \pm j}^{2-}=e^{\left(2 d_{2}^{( \pm j)}-2 \ln b_{2}^{( \pm j)}-c_{22}\right) / 4}$, and $\theta_{2 R}^{-}=\theta_{2 R}+\left(c_{22} / 4\right)$. It is noted that equations (32) and (34) have the similar forms as equation (14). Choosing suitable parameters, we give Figure 5 illustrating the collision between one-peak solitons in the ferromagnetic and polar states. The solitons $S_{1}$ in the ferromagnetic state retain their shapes invariant after the collision, and only has a phase shift at the moment of collision in all components, due to the total spin conservation. However, the amplitudes of one-peak soliton $S_{2}$ in the polar state increase in components $\Phi_{ \pm 2}$ after the collision, accompanied by the decrease of amplitude for $S_{2}$ in component $\Phi_{0}$. For the case of collision between onepeak soliton in the ferromagnetic state and two-peak soliton in the polar state, the collision occurs without change of intensities for the solitons in the ferromagnetic state, while with an intensity redistribution among the twopeak solitons in the polar state in all components, as shown in Figure 6. Therefore, we can conclude that there exists no mixing collision in the internal states for the solitons in the ferromagnetic state outside the collision region.

4.3. Collisions between Two Solitons in the Polar State. In this section, we will consider the collisions between the solitons 


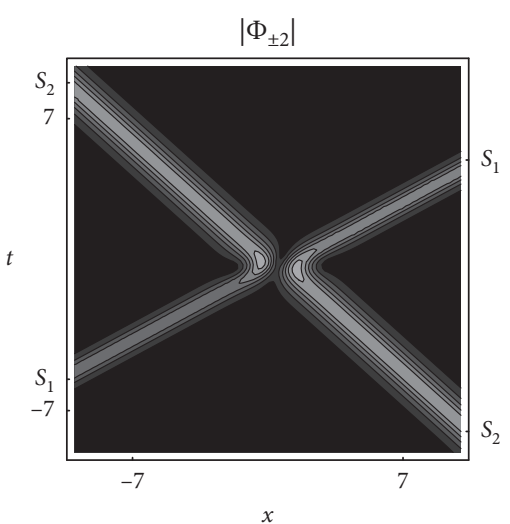

(a)

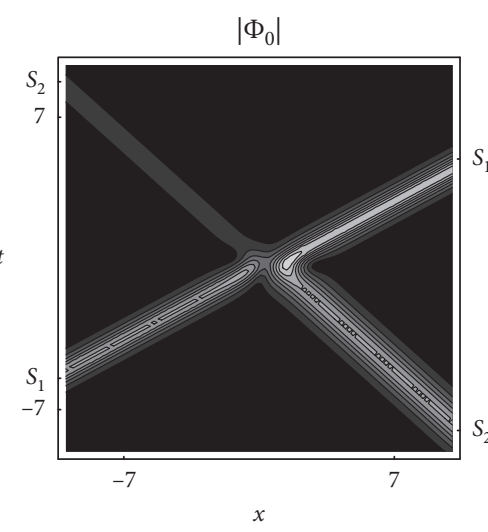

(b)

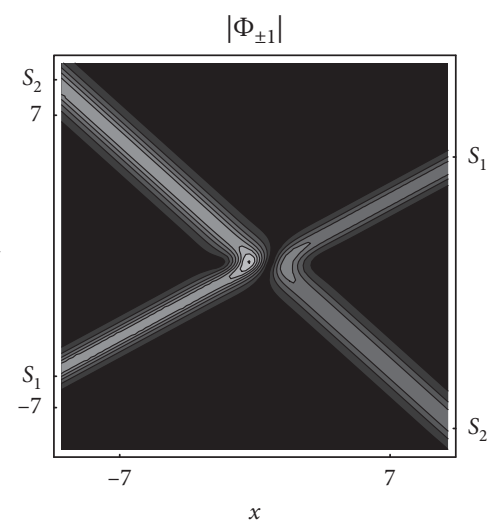

(c)

FIGURE 4: Collision between two solitons in the ferromagnetic state for the components $\Phi_{ \pm j}$ via solution (16). Parameters are $k_{1}=1.2+i$, $k_{2}=1.2-0.6 i, b_{1}^{( \pm 2)}=0.6, b_{1}^{(0)}=\sqrt{0.9}, b_{1}^{( \pm 1)}=0.9, b_{2}^{( \pm 2)}=1.1, b_{2}^{(0)}=\sqrt{0.08}$, and $b_{2}^{( \pm 1)}=\sqrt{1.25}$.

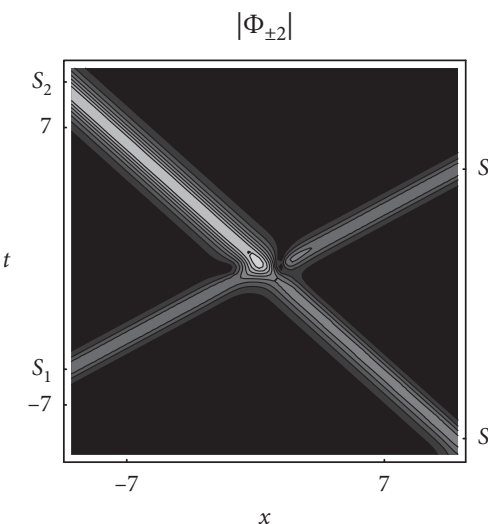

(a)

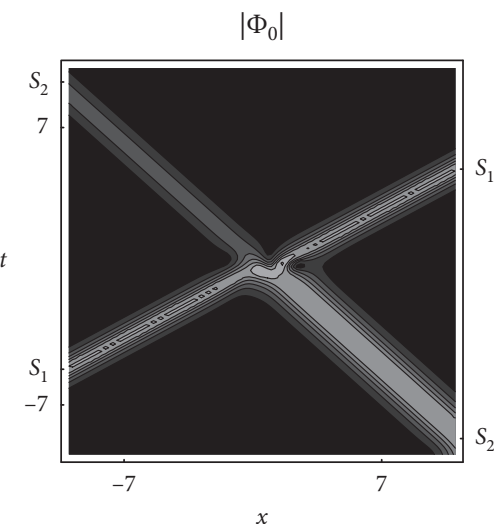

(b)

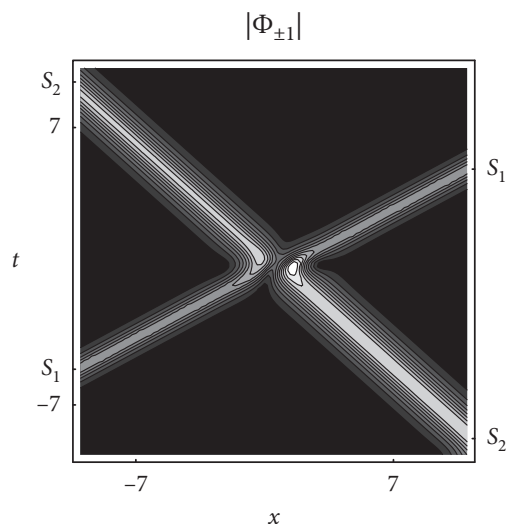

(c)

FIgURE 5: Collision between one-peak solitons in the ferromagnetic and polar states for the components $\Phi_{ \pm j}$ via solution (16). Parameters are the same as those in Figure 4 except $b_{2}^{(2)}=b_{2}^{(-2) *}=0.8+0.5 i, b_{2}^{(0)}=0.3$, and $b_{2}^{(1)}=-b_{2}^{(-1) *}=0.4-0.9 i$.

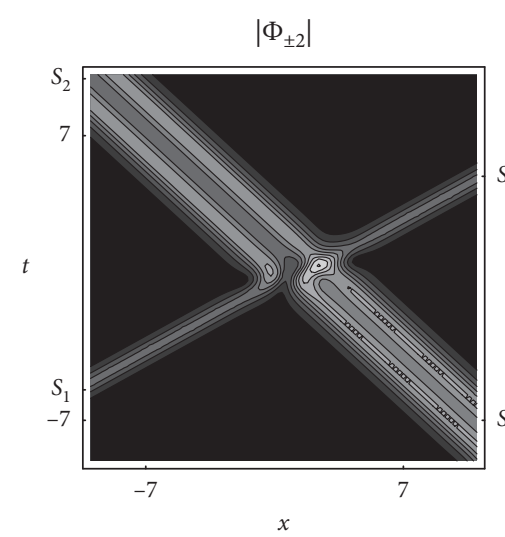

(a)

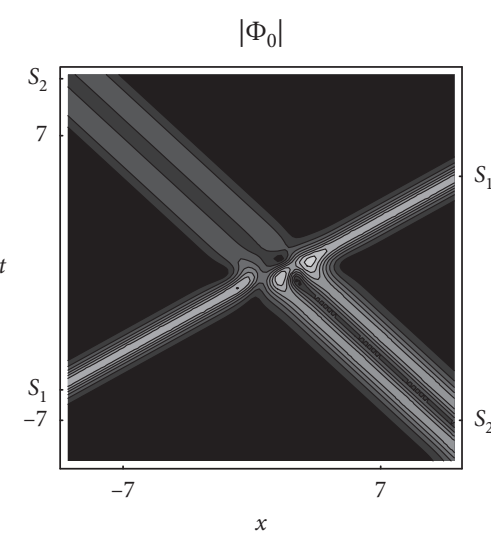

(b)

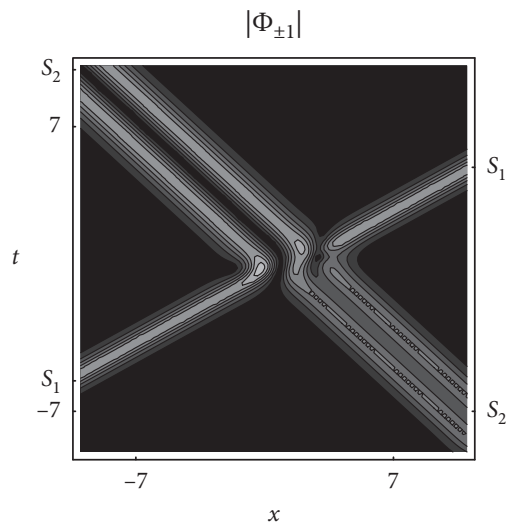

(c)

FIGURE 6: Collision between one-peak soliton in the ferromagnetic state and two-peak soliton in the polar state for the components $\Phi_{ \pm j}$ via solutions (16). Parameters are $k_{1}=1.2+i, k_{2}=1.2-0.6 i, b_{1}^{( \pm 2)}=0.5, b_{1}^{(0)}=1, b_{1}^{( \pm 1)}=\sqrt{0.75}, b_{2}^{( \pm 2)}=0.8, b_{2}^{(0)}=0.4$, and $b_{2}^{( \pm 1)}=\sqrt{0.65}$. 


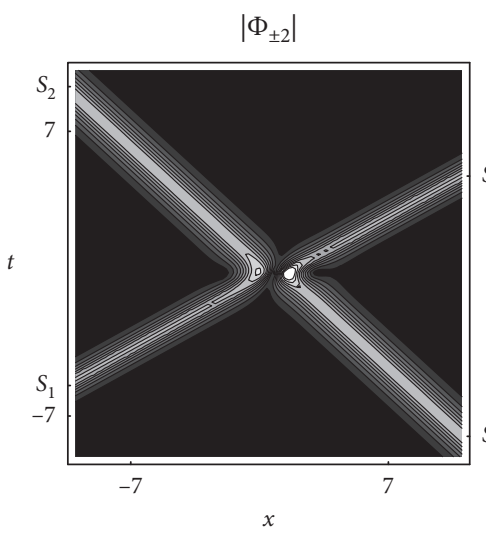

(a)

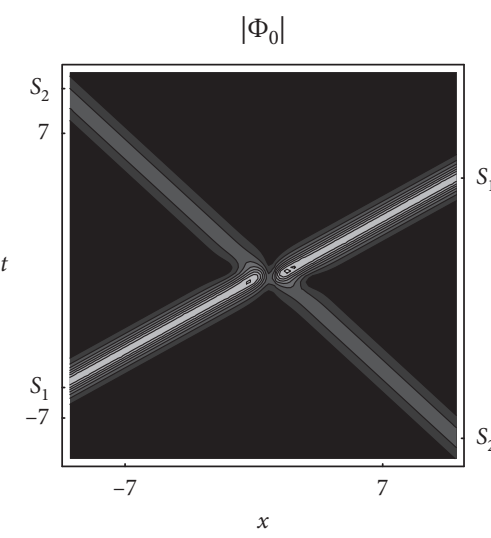

(b)

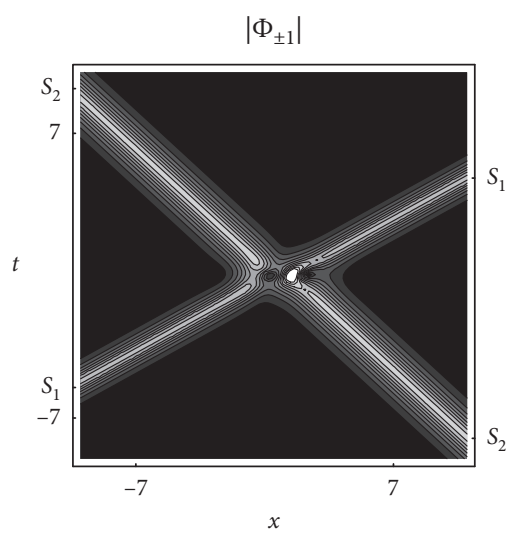

(c)

FIgURE 7: Collision between one-peak solitons in the polar state for the components $\Phi_{ \pm j}$ via solution (16). Parameters are $k_{1}=1.2+i$, $k_{2}=1.2-0.6 i, \quad b_{1}^{(2)}=b_{1}^{(-2) *}=0.5+0.8 i, \quad b_{1}^{(0)}=1.01, \quad b_{1}^{(1)}=-b_{1}^{(-1) *}=0.75-0.6 i, \quad b_{2}^{(2)}=b_{2}^{(-2) *}=0.8+0.5 i, \quad b_{2}^{(0)}=0.3, \quad$ and $\quad b_{2}^{(1)}=-b_{2}^{(-1) *}=$ $0.4-0.9 i$.

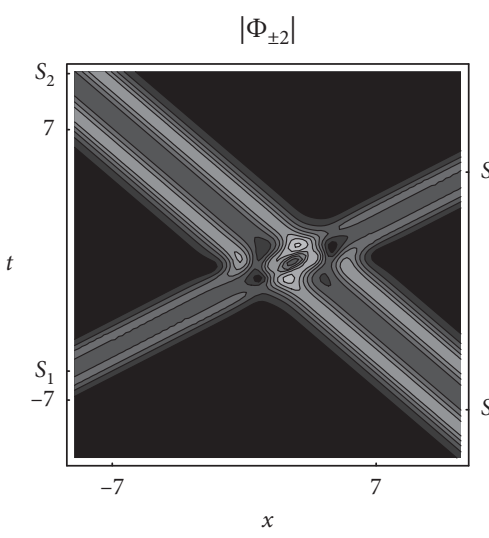

(a)

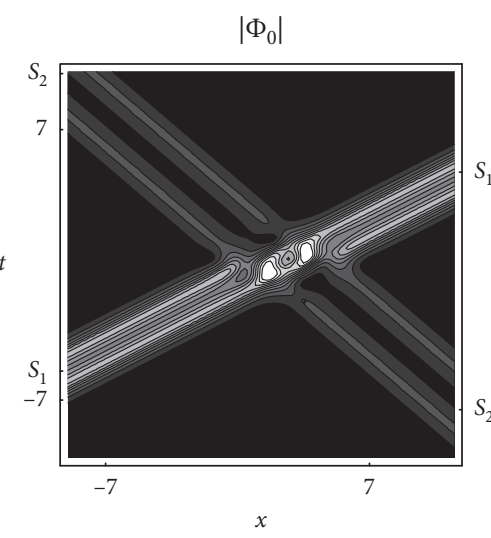

(b)

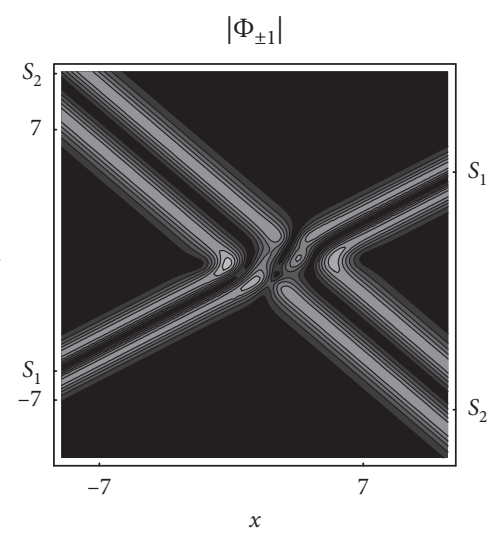

(c)

Figure 8: Collision between two-peak solitons in the polar state for the components $\Phi_{ \pm j}$ via solution (16). Parameters are $k_{1}=1.2+i$, $k_{2}=1.2-0.6 i, b_{1}^{( \pm 2)}=0.5, b_{1}^{(0)}=1.1, b_{1}^{( \pm 1)}=\sqrt{0.75}, b_{2}^{( \pm 2)}=0.8, b_{2}^{(0)}=0.3$, and $b_{2}^{( \pm 1)}=\sqrt{0.66}$.

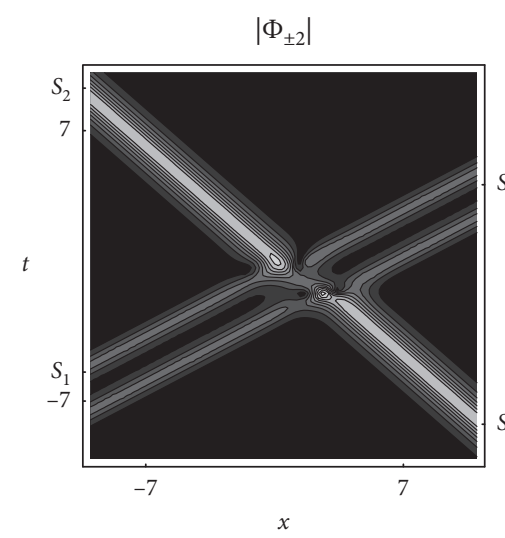

(a)

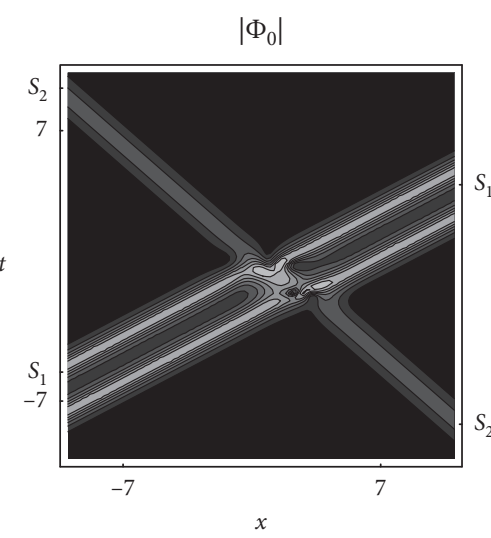

(b)

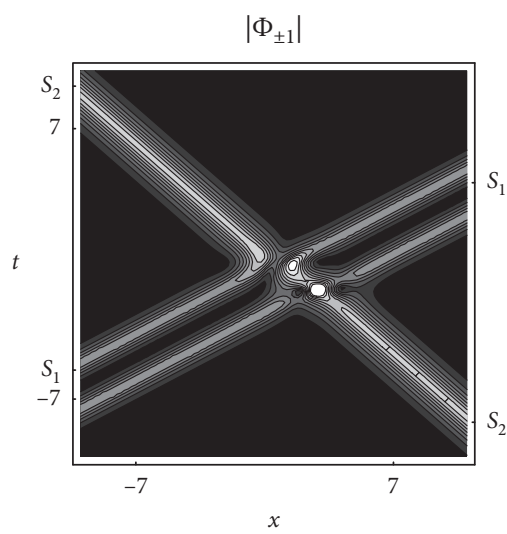

(c)

FIgURE 9: Collision between one- and two-peak solitons in the polar state for the components $\Phi_{ \pm j}$ via solution (16). Parameters are the same as those in Figure 7 except $b_{1}^{( \pm 2)}=0.5, b_{1}^{(0)}=1.01$, and $b_{1}^{( \pm 1)}=\sqrt{0.75}$. 
in the polar state $\left(\Lambda_{m} \neq 0\right)$. In this case, the asymptotic forms are given as

(A) Before the collision.

(1) Soliton $S^{1-}$ :

$$
\Phi_{ \pm j} \longrightarrow A_{ \pm j}^{1-} e^{i \theta_{1 I}} \frac{\cosh \left(\theta_{1 R}^{-}+P_{ \pm j}^{1-}\right)}{4 \cosh ^{2}\left(\theta_{1 R}^{-}\right)+L^{1-}},
$$

where $A_{ \pm j}^{1-}=2 e^{\left(d_{1}^{( \pm j)}+\ln b_{1}^{( \pm j)}-c_{11}\right) / 2}, L^{1-}=e^{\left(l_{1}-\left(c_{11} / 2\right)\right)}-2$, $P_{ \pm j}^{1-}=e^{\left(2 d_{1}^{( \pm j)}-2 \ln b_{1}^{( \pm j)}-c_{11}\right) / 4}$, and $\theta_{1 R}^{-}=\theta_{1 R}+\left(c_{11} / 4\right)$.

(2) Soliton $S^{2-}$ :

$$
\Phi_{ \pm j} \longrightarrow 2 A_{ \pm j}^{2-} e^{i \theta_{2 I}} \frac{\cosh \left(\theta_{2 R}^{-}+P_{ \pm j}^{2-}\right)}{4 \cosh ^{2}\left(\theta_{2 R}^{-}\right)+L^{2-}}
$$

where $\quad A_{ \pm j}^{2-}=2 e^{\left(W_{12}^{( \pm j)}+h_{12}^{( \pm j)}-l_{5}-c_{11}\right) / 2}, \quad L^{2-}=$ $e^{\left(L_{12}-\left(l_{5}+c_{11} / 2\right)\right)}-2, \quad P_{ \pm j}^{2-}=e^{\left(2 W_{12}^{( \pm j)}-2 h_{12}^{( \pm j)}-l_{5}+c_{11}\right) / 4}, \quad$ and $\theta_{2 R}^{-}=\theta_{2 R}+\left(\left(l_{5}-c_{11}\right) / 4\right)$.

(B) After the collision.

(1) Soliton $S^{1+}$ :

$$
\Phi_{ \pm j} \longrightarrow 2 A_{ \pm j}^{1+} e^{i \theta_{1 I}} \frac{\cosh \left(\theta_{1 R}^{+}+P_{ \pm j}^{1+}\right)}{4 \cosh ^{2}\left(\theta_{1 R}^{+}\right)+L^{1+}},
$$

where $\quad A_{ \pm j}^{1+}=2 e^{\left(W_{21}^{( \pm j)}+h_{21}^{( \pm j)}-l_{5}-c_{22}\right) / 2}=\left(\left(\left(k_{1}-k_{2}\right)\left(k_{1}^{*}+\right.\right.\right.$ $\left.\left.\left.k_{2}\right)\right) /\left(\left(k_{1}^{*}-k_{2}^{*}\right)\left(k_{1}+k_{2}^{*}\right)\right)\right) A_{ \pm j}^{1-}, L^{1+}=e^{L_{21}-\left(\left(l_{5}+c_{22}\right) / 2\right)}-2$, $\theta_{1 R}^{+}=\theta_{1 R}+\left(\left(l_{5}-c_{22}\right) / 4\right), \quad$ and $\quad P_{ \pm j}^{1+}=$ $e^{\left(2 W_{21}^{( \pm j)}-2 h_{21}^{( \pm j)}-l_{5}+c_{22}\right) / 4}$.

(2) Soliton $S^{2+}$ :

$$
\Phi_{ \pm j} \longrightarrow 2 A_{ \pm j}^{2+} e^{i \theta_{2 I}} \frac{\cosh \left(\theta_{2 R}^{+}+P_{ \pm j}^{2+}\right)}{4 \cosh ^{2}\left(\theta_{2 R}^{+}\right)+L^{2+}},
$$

where $A_{ \pm j}^{2+}=2 e^{\left(d_{2}^{( \pm j)}+\ln b_{2}^{( \pm j)}-c_{22}\right) / 2}=\left(\left(\left(k_{1}^{*}-k_{2}^{*}\right)\left(k_{1}^{*}+k_{2}\right)\right) /\right.$ $\left.\left(k_{1}-k_{2}\right)\left(k_{1}+k_{2}^{*}\right)\right) A_{ \pm j}^{2-}, L^{2+}=e^{\left(l_{2}-\left(c_{22} / 2\right)\right)}-2, \quad P_{ \pm j}^{2+}=$ $e^{\left(2 d_{2}^{( \pm j)}-2 \ln b_{2}^{( \pm j)}-c_{22}\right) / 4}$, and $\theta_{2 R}^{+}=\theta_{2 R}+\left(c_{22} / 4\right)$. Figures 7-9, respectively, describe the collisions between two onepeak solitons, two two-peak solitons, and one-peak and two-peak solitons in the polar state. From equations (32)-(35), one can find that the collisions are elastic in this case. As shown in Figures 7-9, solitons with one or two peaks collide with each other and the intensity and shape of each soliton keeps invariant after the collision.

\section{Conclusions}

In this paper, we have investigated the solitons and their collisions for the five-component GP equations, i.e., equation (1), which can describe the dynamics of an $F=2$ spinor BECs in one dimension. Through the Hirota method and symbolic computation, we have derived one-soliton solutions (8) and two-soliton solutions (16) for equation (1). With different polarization parameters and spin densities, one-soliton solutions (8) have been classified as (a) the one-peak soliton in the ferromagnetic and cyclic states (as seen in Figures 1 and 2); (b) one- and two-peak solitons in the polar state (as seen in Figure 3). For the two-peak soliton in the polar state, the distance between the two peaks increases inversely with the polarization parameter $\Lambda_{1}$ (as seen in Figure 3). Performing the asymptotic analysis on two-soliton solutions (16), we have analyzed the collisions between two solitons in the same and different states. For the collisions between two solitons in the ferromagnetic state, we have shown the constrained condition, under which the collision is elastic. In addition, we have given the example of the inelastic collision with intensity redistribution (as seen in Figure 4), and it is feasible to exploit the inelastic collision in the design of soliton switching architectures. For the collisions between soliton in the ferromagnetic state and solitons in the polar state, the solitons in the ferromagnetic state keep their characteristics invariant while the shapes and intensities change for those in the polar state after the collision (as seen in Figures 5 and 6). For the collisions between two solitons in the polar state, the procedure occurs only with a phase shift for each soliton at the moment of collision, and the collisions are inelastic (as seen in Figures 7-9). We hope that the results given in this paper will be of value for the applications in the atom laser and coherent atom transport, which can contribute to the realization of quantum information processors or computation.

\section{Data Availability}

The data used to support the findings of this study are available from the corresponding author upon request.

\section{Conflicts of Interest}

The authors declare that they have no conflicts of interest.

\section{Acknowledgments}

This work was supported by the National Natural Science Foundation of China under Grant nos. 11501526 and 11871232, Key Projects of Science and Technology Research of the Henan Education Department, (No. 17A110035), and Doctoral Research Fund of Zhengzhou University of Light Industry.

\section{References}

[1] K. E. Strecker, G. B. Partridge, A. G. Truscott, and R. G. Hulet, "Formation and propagation of matter-wave soliton trains," Nature, vol. 417, no. 6885, pp. 150-153, 2002.

[2] L. Khaykovich, F. Schreck, G. Ferrari et al., "Formation of a matter-wave bright soliton," Science, vol. 296, p. 1290, 2002.

[3] C. J. Pethick and H. Smith, Bose-Einstein Condensation in Dilute Gases, Cambridge University, Cambridge, UK, 2008.

[4] P. G. Kevrekidis, D. J. Frantzeskakis, and R. CarreteroGonzález, Emergent Nolinear Phenomena in Bose-Einstein Condensates: Theory and Experiment, Springer, Berlin, Germany, 2008.

[5] B. Xiong and J. B. Gong, "Dynamical creation of complex vector solitons in spinor Bose-Einstein condensates," Physical Review A, vol. 81, p. 033618, 2010. 
[6] E. V. Doktorov, V. M. Rothos, and Y. S. Kivshar, "Full-time dynamics of modulational instability in spinor Bose-Einstein condensates," Physical Review A, vol. 76, p. 013626, 2007.

[7] T.-L. Ho, "Spinor Bose condensates in optical traps," Physical Review Letters, vol. 81, no. 4, pp. 742-745, 1998.

[8] T. Ohmi and K. Machida, "Bose-Einstein condensation with internal degrees of freedom in alkali atom gases," Journal of the Physical Society of Japan, vol. 67, p. 1882, 1998.

[9] J. Stenger, S. Inouye, D. M. Stamper-Kurn, H.-J. Miesner, A. P. Chikkatur, and W. Ketterle, "Spin domains in groundstate Bose-Einstein condensates," Nature, vol. 396, no. 6709, pp. 345-348, 1998.

[10] M. Ueda and M. Koashi, "Theory of spin-2 Bose-Einstein condensates: spin correlations, magnetic response, and excitation spectra," Physical Review A, vol. 65, p. 063602, 2002.

[11] M.-S. Chang, C. D. Hamley, M. D. Barrett et al., "Observation of spinor dynamics in optically trapped Rb87 Bose-Einstein condensates," Physical Review Letters, vol. 92, p. 140403, 2004.

[12] H. Schmaljohann, M. Erhard, J. Kronjäger et al., "Dynamics of $F=2$ spinor Bose-Einstein condensates," Physical Review Letters, vol. 92, p. 040402, 2004.

[13] P. Rosenbusch, V. Bretin, and J. Dalibard, "Dynamics of a single vortex line in a Bose-Einstein condensate," Physical Review Letters, vol. 89, p. 200403, 2002.

[14] L. D. Carr and J. Brand, "Spontaneous soliton formation and modulational instability in Bose-Einstein condensates," Physical Review Letters, vol. 92, p. 040401, 2004.

[15] C. M. Jiang, A. Zada, T. Senel, and T. X. Li, "Synchronization of bidirectional $\mathrm{N}$-coupled fractional-order chaotic systems with ring connection based on antisymmetric structure," Advances in Difference Equations, vol. 2019, 456, 2019.

[16] A. Yang, L. Li, Z. Wang, and R. Guo, "Tracking control of a class of chaotic systems," Symmetry, vol. 11, no. 4, p. 568, 2019.

[17] R. Gao, J. Xu, and H. Zhang, "Receding horizon control for multiplicative noise stochastic systems with input delay," Automatica, vol. 81, pp. 390-396, 2017.

[18] R. Gao, J. Xu, W. Li, and X. Liu, "A necessary and sufficient RHC stabilizability condition for stochastic control with delayed input," Applied Mathematics and Computation, vol. 360, pp. 122-130, 2019.

[19] R. Gao, X. Liu, and H. Zhang, "Receding horizon control for discrete-time multiple input delay systems," Optimal Control Applications and Methods, vol. 38, no. 6, pp. 1187-1192, 2017.

[20] A. Hasegawa and F. Tappert, "Transmission of stationary nonlinear optical pulses in dispersive dielectric fibers. I. Anomalous dispersion," Applied Physics Letters, vol. 23, no. 3, pp. 142-144, 1973.

[21] A. Hasegawa and M. Matsumoto, Optical Solitons in Fibers, Springer, Berlin, Germany, 2003.

[22] K. E. Lonngren, "Soliton experiments in plasmas," Plasma Physics, vol. 25, no. 9, pp. 943-982, 1983.

[23] L. Li, Z. Li, B. A. Malomed, D. Mihalache, and W. M. Liu, "Exact soliton solutions and nonlinear modulation instability in spinor Bose-Einstein condensates," Physical Review A, vol. 72, p. 033611, 2005.

[24] Z. D. Li, Q. Y. Li, L. Li, and W. M. Liu, "Soliton solution for the spin current in a ferromagnetic nanowire," Physical Review E, vol. 76, p. 026605, 2007.

[25] Z. D. Li, Q. Y. Li, T. F. Xu, and P. B. He, "Publisher's Note: breathers and rogue waves excited by all-magnonic spintransfer torque [Phys. Rev. E 94, 042220 (2016)]," Physical Review E, vol. 94, p. 042220, 2016.

[26] P. Meystre, Atom Optics, Springer, Berlin, Germany, 2001.
[27] Z. D. Li, P. B. He, L. Li, J. Q. Liang, and W. M. Liu, "Magnetic soliton and soliton collisions of spinor Bose-Einstein condensates in an optical lattice," Physical Review A, vol. 71, p. 053611, 2005.

[28] T. F. Xu, W. L. Li, Z.-D. Li, and C. Zhang, "Phase diagram and dynamics of dark-bright vector solitons in spin-orbit-coupled Bose-Einstein condensate," Chaos, Solitons \& Fractals, vol. 111, pp. 62-67, 2018.

[29] Z.-D. Li and Q.-Y. Li, "Dark soliton interaction of spinor Bose-Einstein condensates in an optical lattice," Annals of Physics, vol. 322, no. 8, pp. 1961-1971, 2007.

[30] Z.-D. Li, Q.-Y. Li, X.-H. Hu, Z.-X. Zheng, and Y. Sun, "Hirota method for the nonlinear Schrödinger equation with an arbitrary linear time-dependent potential," Annals of Physics, vol. 322, no. 11, pp. 2545-2553, 2007.

[31] M. Uchiyama, J. i. Ieda, and M. Wadati, "Multicomponent bright solitons in $F=2$ spinor bose-einstein condensates," Journal of the Physical Society of Japan, vol. 76, no. 7, p. $074005,2007$.

[32] V. S. Gerdjikov, N. A. Kostov, and T. I. Valchev, "Solutions of multi-component NLS models and Spinor Bose-Einstein condensates," Physica D: Nonlinear Phenomena, vol. 238, no. 15 , pp. 1306-1310, 2009.

[33] C. V. Ciobanu, S.-K. Yip, and T.-L. Ho, "Phase diagrams of $F=2$ spinor Bose-Einstein condensates," Physical Review A, vol. 61, p. 033607, 2000.

[34] B. Tian, Y.-T. Gao, and H.-W. Zhu, "Variable-coefficient higher-order nonlinear Schrödinger model in optical fibers: variable-coefficient bilinear form, Bäcklund transformation, brightons and symbolic computation," Physics Letters A, vol. 366, no. 3, pp. 223-229, 2007.

[35] B. Tian and Y.-T. Gao, "Variable-coefficient higher-order nonlinear Schrödinger model in optical fibers: new transformation with burstons, brightons and symbolic computation," Physics Letters A, vol. 359, no. 3, pp. 241-248, 2006.

[36] R. Hirota, The Direct Method in Soliton Theory, Cambridge University, Cambridge, UK, 2004.

[37] R. Hirota, "Exact envelope-soliton solutions of a nonlinear wave equation," Journal of Mathematical Physics, vol. 14, no. 7, pp. 805-809, 1973.

[38] J. Ieda, T. Miyakawa, and M. Wadati, "Exact analysis of soliton dynamics in spinor Bose-Einstein condensates," Physical Review Letters, vol. 93, p. 194102, 2004.

[39] J. Babarro, M. J. Paz-Alonso, H. Michinel, J. R. Salgueiro, and D. N. Olivieri, "Controllable scattering of vector Bose-Einstein solitons," Physical Review A, vol. 71, p. 043608, 2005.

[40] H. Q. Zhang, X. H. Meng, T. Xu, L. L. Li, and B. Tian, "Integrability of an $\mathrm{N}$-coupled nonlinear Schrödinger system for polarized optical waves in an isotropic medium via symbolic computation," Physical Review E, vol. 77, p. 026605, 2008. 